Ergod. Th. \& Dynam. Sys. (2020), 40, 992-1007 C Cambridge University Press, 2018

This is an Open Access article, distributed under the terms of the Creative Commons Attribution licence (http://creativecommons.org/licenses/by/4.0/), which permits unrestricted re-use, distribution, and reproduction in any medium, provided the original work is properly cited.

doi:10.1017/etds.2018.53

\title{
On uniform distribution of polynomials and good universality
}

\author{
RADHAKRISHNAN NAIR and ENTESAR NASR \\ Mathematical Sciences, University of Liverpool, Liverpool L69 7ZL, UK \\ (e-mail:nair@liv.ac.uk,hsenasr@liv.ac.uk)
}

(Received 13 August 2017 and accepted in revised form 6 June 2018)

Dedicated to the memory of Klaus Roth

Abstract. Suppose $\left(k_{n}\right)_{n \geq 1}$ is Hartman uniformly distributed and good universal. Also suppose $\psi$ is a polynomial with at least one coefficient other than $\psi(0)$ an irrational number. We adapt an argument due to Furstenberg to prove that the sequence $\left(\psi\left(k_{n}\right)\right)_{n \geq 1}$ is uniformly distributed modulo one. This is used to give some new families of Poincaré recurrent sequences. In addition we show these sequences are also intersective and Glasner.

\section{Introduction}

Let $(X, \mathcal{B}, \mu)$ be a probability space and let $T: X \rightarrow X$ be a measurable map, that is also measure-preserving. That is, given $A \in \mathcal{B}$, we have $\mu\left(T^{-1} A\right)=\mu(A)$, where $T^{-1} A$ denotes the set $\{x \in X: T x \in A\}$. We call $(X, \mathcal{B}, \mu, T)$ a dynamical system. We say that the dynamical system is ergodic if $T^{-1} A=A$ for $A \in \mathcal{B}$ means that either $\mu(A)$ or $\mu(X \backslash A)$ is 0 .

We say that $\left(k_{n}\right)_{n \geq 1} \subseteq \mathbb{N}$ is $L^{p}$ good universal if for each dynamical system $(X, \mathcal{B}, \mu, T)$ and for each $f \in L^{p}(X, \beta, \mu)$ the limit

$$
\ell_{T, f}(x)=\lim _{N \rightarrow \infty} \frac{1}{N} \sum_{n=0}^{N-1} f\left(T^{k_{n}} x\right)
$$

exists $\mu$ almost everywhere.

In what follows we will call $\left(k_{n}\right)_{n \geq 1}$ good if it is $L^{\infty}$-good universal and we have $\ell_{T, f}(T x)=\ell_{T, f}(x) \mu$ almost everywhere.

We say that a sequence $x_{1}, \ldots, x_{N}, \ldots$ is uniformly distributed modulo one if

$$
\lim _{N \rightarrow \infty} \frac{1}{N} \#\left\{1 \leq n \leq N:\left\{x_{n}\right\} \in I\right\}=|I|
$$


for every interval $I \subseteq[0,1)$. For a real number $y$ we have used $\{y\}$ to denote its fractional part and let $[y]=y-\{y\}$ denote its integer part. In this paper we prove the following theorem.

THEOREM 1.1. Let $\psi(x)=\alpha_{0}+\alpha_{1} x+\alpha_{2} x^{2}+\cdots+\alpha_{l} x^{l}$, with at least one of the numbers $\alpha_{1}, \ldots, \alpha_{l}$ irrational. Then the sequence $\left(\psi\left(k_{n}\right)\right)_{n \geq 1}$ is uniformly distributed modulo one if $\left(k_{n}\right)_{n \geq 1}$ is good.

The theorem is proved using Furstenberg's skew product method [15]. This seems to be the first new family of uniformly distributed sequences provided by this method. Theorem 1.1 has a number of corollaries. For $f \in L^{p}(X, \mathcal{B}, \mu)$, with $p \geq 1$, set $\|f\|_{p}=$ $\left(\int_{X}|f|^{p} d \mu\right)^{1 / p}$.

COROLlARY 1.2. Suppose that $\phi$ is a non-constant polynomial, mapping the natural numbers to themselves, and that $\left(k_{n}\right)_{n \geq 1}$ is good. Then for any dynamical system $(X, \mathcal{B}, \mu, T)$ and any $f \in L^{2}(X, \mathcal{B}, \mu)$, there exists $\bar{f} \in L^{2}(X, \mathcal{B}, \mu)$ such that

$$
\lim _{N \rightarrow \infty}\left\|\frac{1}{N} \sum_{n=1}^{N} f\left(T^{\phi\left(k_{n}\right)} x\right)-\bar{f}\right\|_{2}=0 .
$$

It is natural to ask if Corollary 1.2 is true almost everywhere.

We say that a set of natural numbers $S$ has positive Banach density $B(S)$ if there exists a sequence of intervals $\left(I_{p}\right)_{p=1}^{\infty}$ in $\mathbb{N}$ with $I_{p}=\left[a_{p}, b_{p}\right] \cap \mathbb{N}$ and $\left|I_{p}\right|=b_{p}-a_{p}$ tending to infinity as $p$ tends to infinity such that

$$
\lim _{p \rightarrow \infty} \frac{\left|S \cap I_{p}\right|}{\left|I_{p}\right|}=B(S),
$$

and for any other sequence of intervals $\left(I_{p}^{\prime}\right)_{p=1}^{\infty}$ in $\mathbb{N}$ such that $\left|I_{p}^{\prime}\right|$ tends to infinity as $p$ tends to infinity we have

$$
\limsup _{p \rightarrow \infty} \frac{\left|S \cap I_{p}^{\prime}\right|}{\left|I_{p}^{\prime}\right|} \leq B(S) .
$$

We say that a sequence $\kappa=\left(k_{n}\right)_{n=1}^{\infty}$ of positive integers is a set of intersectivity if, given any set of natural numbers $S$ of positive Banach density $B(S)$, there exists an integer $k$ in $\kappa$ such that we can find $s_{1}$ and $s_{2}$ both in $S$ satisfying

$$
k=s_{1}-s_{2} .
$$

Following [16], we say that a sequence of natural numbers $\kappa=\left(k_{n}\right)_{n=1}^{\infty}$ is Poincaré recurrent if, given any dynamical system on a probability space $(X, \beta, \mu, T)$ and any set $A$ in $\beta$ of positive measure, there exists an element $k$ of $\kappa$ such that

$$
\mu\left(A \cap T^{-k} A\right)>0 .
$$

We say that a sequence of natural numbers $\kappa=\left(k_{n}\right)_{n=1}^{\infty}$ is strongly Poincaré recurrent if, given any dynamical system on a probability space $(X, \beta, \mu, T)$ and any set $A$ in $\beta$ of positive measure, there exist $\gamma_{\kappa, A}>0$ and an element $k$ of $\kappa$ such that

$$
\mu\left(A \cap T^{-k} A\right) \geq \gamma_{\kappa}, A .
$$


COROLLARY 1.3. Suppose $\phi$ is a non-constant polynomial mapping the natural numbers to themselves such that $\phi(0)=0$. Then the sequence $\left(\phi\left(k_{n}\right)\right)_{n \geq 1}$ is strongly recurrent if $\left(k_{n}\right)_{n \geq 1}$ is good.

Poincaré recurrent sequences are the same as intersective sequences [9], hence we have the following corollary.

COROLLARY 1.4. Suppose $\phi$ is a non-constant polynomial mapping the natural numbers to themselves such that $\phi(0)=0$. Then the sequence $\left(\phi\left(k_{n}\right)\right)_{n \geq 1}$ is intersective if $\left(k_{n}\right)_{n \geq 1}$ is good.

It is natural to ask if the sequences from Corollaries 1.3 and 1.4 are also multiply recurrent and multiply intersective.

Following [5], we say that a set $S$ contained in $\mathbb{Z}$ is Glasner if for every infinite set $Y$ contained in $\mathbb{T}=\mathbb{R} / \mathbb{Z}$ and $\epsilon>0$, some dilation $n Y=\{n y: y \in Y\}$ is $\epsilon$ dense (that is, $n Y$ intersects every open interval of length $\epsilon$ ). This definition is motivated by the 1979 result of Glasner [17] which says that, given an infinite set $Y \subset \mathbb{T}$, there exists a natural number $n$ such that $n Y$ is $\epsilon$ dense in $\mathbb{T}$.

We have the following theorem, which in the case $k_{n}=n(n=1,2, \ldots)$, appears in [2].

COROLLARY 1.5. Let $\phi$ be a polynomial of degree $l \geq 1$ mapping the natural numbers to themselves. Suppose $\delta>0$. Then, if $\left(k_{n}\right)_{n \geq 1}$ is good, there exists $\epsilon(\phi, \delta)>0$ such that if $0<\epsilon<\epsilon(\phi, \delta)$ for any set $Y$ contained in $\mathbb{T}$ of cardinality s, where

$$
s>\left(\frac{1}{\epsilon}\right)^{2 l+\delta},
$$

we have an $\epsilon$ dense dilation of the form $\phi\left(k_{n}\right) Y$, for some natural number $n$.

Since the proof of Weyl's theorem [39], now over a century ago, innumerable examples of uniformly distributed sequences have been produced. See the standard references [14] and [23] for more background. Despite its age, Weyl's theorem remains of interest, in part because of its applications. Most proofs of Weyl's theorem rely on exponential sums. An ergodic-theoretic proof of Weyl's theorem, making no use of exponential sums, was given by Furstenberg [15]. This is the point of view taken in this paper. That said, exponential sums will also play a role in our considerations.

Inspired by Roth's proof that a set of integers of positive density contains a threeterm arithmetic progression [37, Ch. 10], Sárközy [31] showed that any set of integers of positive density contains two terms whose difference is a square. Later, in stages, he also considered more general polynomials than squares and also primes minus one $[\mathbf{3 2}, \mathbf{3 3}]$.

Soon thereafter Furstenberg used Weyl's theorem and an ergodic method to prove that if $P(x)$ is a non-constant polynomial with integer coefficients such that $P(0)=0$ then $(P(n))_{n \geq 1}$ is a set of recurrence [16].

Let $\left(p_{n}\right)_{n \geq 1}$ denote the sequence of prime numbers. In [24] polynomials $P$ such that $\left(P\left(p_{n}\right)\right)_{n \geq 1}$ and $(P(n))_{n \geq 1}$ are sets of strong recurrence are fully classified. The primary tools used to prove the first author's single recurrence results are a method of Furstenberg, together with the uniform distribution of the sequences $\left(\psi\left(p_{n}\right)\right)_{n \geq 1}$ and $(\psi(n))_{n \geq 1}$. The extension of these results to $\mathbb{Z}^{r}$ for $r \in \mathbb{N}$ rather than just $\mathbb{Z}$ is routine and for this reason not discussed in either [24] or [26]. The modifications amount to working with characters 
of $\mathbb{Z}^{r}$ rather than $\mathbb{Z}$ which work very similarly. In [8] it is shown that if $\eta$ is a polynomial with integer coefficients mapping the natural numbers to themselves, then $\left(\eta\left(p_{n}-1\right)\right)_{n \geq 1}$ is strongly Poincaré recurrent. Evidently this is just a special case of [24, Theorem 1]. There is now of course a literature on the multiple recurrence of these sequences, too extensive to conveniently discuss here.

A sequence in a second countable locally compact group is called Hartman uniformly distributed if it is uniformly distributed on the Bohr compactification of the locally compact group. In [26] it is shown that any such sequence is strongly recurrent. In the special cases of $\mathbb{Z}$ and $\mathbb{R}$ this observation is anticipated in [1] and [11]. As is shown in [26], one can use Weyl's criterion on the Bohr compactification to construct examples. In the particular case of $\mathbb{Z}$, the methods of analytic number theory are used to construct a number of families of strongly Poincaré recurrent sequences. See [25, Theorem 4] for details of this. Let $\left(\alpha_{1}, \ldots, \alpha_{r}\right)$ be an $r$-tuple of non-integer real numbers all greater than 1 . Then $\left(\left[p_{n}^{\alpha_{1}}\right], \ldots,\left[p_{n}^{\alpha_{r}}\right]\right)_{n \geq 1}$ is shown in [6] to be strongly Poincaré recurrent. In fact, while not discussed in [26], the sequence $\left(\left[p_{n}^{\alpha_{1}}\right], \ldots,\left[p_{n}^{\alpha_{r}}\right]\right)_{n \geq 1}$ is Hartman uniformly distributed on $\mathbb{Z}^{r}$. Therefore the fact that it is strongly Poincaré recurrent follows from [26, Theorem 1].

In [22] we adapt the ideas of Furstenberg to study the Poincaré recurrence-intersectivity phenomenon in positive characteristic. In this context the analogue of the integers is the ring of polynomials $\mathbb{F}_{q}[x]$ over the finite field $\mathbb{F}_{q}$ in characteristic $q$. We show in [22] that the set of irreducibles in $\mathbb{F}_{q}[x]$, which is the analogue of the primes in $\mathbb{Z}$, once shifted by one, is both a set of strong recurrence and a set of intersectivity. Poincaré recurrent sets are intersective sets in this context. Whether the converse is true is unknown to the authors. In [22] it is shown that if $q=p^{r}$ for some natural number $r$ and exceeds the degree of a polynomial $P$ over $\mathbb{F}_{q}$ then $\left\{P(x): x \in \mathbb{F}_{q}\right\}$ is also both a set of strong recurrence and a set of intersectivity. Later in [7] this restriction on degree is removed, by proving the analogue of Weyl's theorem in positive characteristic. This is done by using Van der Corput instead of Weyl differencing.

In [2] it is shown that if $P$ is a non-constant polynomial mapping the natural numbers to themselves, then the sets $\{P(n): n \in \mathbb{N}\}$ and $\left\{p_{n}: n \in \mathbb{N}\right\}$, are Glasner. In [2] there is also a consideration of the quantitative forms of results. The methods in [2] are Fourier analytic and this is what allows these more quantitative forms of the result. See also [5] for earlier results. In [28] it is shown that $\left\{P\left(p_{n}\right): n \in \mathbb{N}\right\}$ is Glasner and in [20] it is shown that any sequence that is Hartman uniformly distributed on $\mathbb{Z}$ is again Glasner. Here again, the methods are Fourier analytic and the theorems are quantitative.

\section{Proof of Theorem 1.1}

We say that a sequence of integers is uniformly distributed on $\mathbb{Z}$ if it is uniformly distributed among the residue classes modulo $m$, for each natural number $m>1$. We say that a sequence of natural numbers $\left(k_{n}\right)_{n \geq 1}$ is Hartman uniform distributed (on $\mathbb{Z}$ ) if it is uniformly distributed on $\mathbb{Z}$, and for each irrational number $\alpha$ the sequence $\left(\left\{k_{n} \alpha\right\}\right)_{n \geq 1}$ is uniformly distributed modulo one. This condition coincides with $\left(k_{n}\right)_{n \geq 1}$ being uniformly distributed on the Bohr compactification of $\mathbb{Z}$. Note that if $\left(k_{n}\right)_{n \geq 1}$ is Hartman uniformly distributed on $\mathbb{Z}$, and if for $z$ with $|z|=1$ we set

$$
F(N, z):=\frac{1}{N} \sum_{n=0}^{N-1} z^{k_{n}} \quad(N=1,2, \ldots),
$$


then

$$
\lim _{N \rightarrow \infty} F(N, z)= \begin{cases}1 & \text { if } z=1, \\ 0 & \text { if } z \text { otherwise. }\end{cases}
$$

The converse is also true. See [23, Example 5.11 on p. 296]. In what follows, when we say that a sequence is Hartman uniformly distributed we mean it is Hartman uniformly distributed on $\mathbb{Z}$.

The first lemma is [10, Corollary 3].

LEMMA 2.1. The following are equivalent.

(i) $\quad\left(k_{n}\right)_{n \geq 1}$ is Hartman uniformly distributed.

(ii) For any dynamical system $(X, \mathcal{B}, \mu, T)$ and $f \in L^{2}(X, \mathcal{B}, \mu)$, if $P_{T} f$ denotes the projection of $f$ onto the $T$ invariant subspace of $L^{2}(X, \mathcal{B}, \mu)$ we have

$$
\lim _{N \rightarrow \infty}\left\|\frac{1}{N} \sum_{n=0}^{N-1} f\left(T^{k_{n}} x\right)-P_{T} f\right\|_{2}=0 .
$$

The next lemma is a special case of a theorem due to Sawyer [34].

Lemma 2.2. Suppose for a dynamical system $(X, \beta, \mu, T)$ that $f \in L^{q}(X, \beta, \mu)$ for some $q \in(1,2)$. Set

$$
M f(x)=\sup _{N \geq 1}\left|\frac{1}{N} \sum_{n=1}^{N} f\left(T^{k_{n}} x\right)\right| \quad(N=1,2, \ldots) .
$$

If $\left(k_{n}\right)_{n \geq 1}$ is $L^{q}$-good universal, then there exists $C>0$ such that $\|M f\|_{2} \leq C\|f\|_{2}$.

A central tool of ours is the following lemma.

LEMmA 2.3. Suppose $\left(k_{i}\right)_{i=1}^{\infty}$ is $L^{q}$-good universal for $q \in(1,2]$. Then if $\left(k_{i}\right)_{i=1}^{\infty}$ is Hartman uniformly distributed and $f \in L^{2}(X, \beta, \mu, T)$, we have $\ell_{T, f}(T x)=\ell_{T, f}(x)$ $\mu$ almost everywhere for any dynamical system $(X, \beta, \mu)$. Further, we have $\ell_{T, f}(x)=$ $\int_{X} f d \mu, \mu$ almost everywhere if $(X, \beta, \mu, T)$ is ergodic.

The sequence $k_{n}=n^{2}(n=1,2, \ldots)$ is $L^{p} \operatorname{good}$ universal for all $p>1$ [3] but not Hartman uniformly distributed. To see this, note that a square integer is never congruent to 3 modulo 4. If we take $X=\mathbb{Z}_{p}$-the $p$-adic integers, set $\mathcal{B}$ to be the Haar $\sigma$-algebra on $\mathbb{Z}_{p}$, set $\mu$ to be Haar measure on $\mathbb{Z}_{p}$ and define $T: \mathbb{Z}_{p} \rightarrow \mathbb{Z}_{p}$ by $T x=x+1$, the dynamical system we obtain is the $p$-adic adding machine, which is ergodic. For $f \in L^{p}\left(\mathbb{Z}_{p}\right)$ it is possible using Fourier analysis to calculate

$$
\lim _{N \rightarrow \infty} \frac{1}{N} \sum_{n=1}^{N} f\left(x+n^{2}\right)
$$

almost everywhere, which we know must exist because $k_{n}=n^{2}(n=1,2, \ldots)$ is $L^{p}$ good universal. The limit is, however, not the Haar integral of $f$ on $\mathbb{Z}_{p}$ as you might expect, but rather a more complicated expression involving Fourier multipliers and Gauss sums. See [3] for these details. This means that for the limit $\ell_{T, f}(x)$ for squares to be the integral of $f$ we need more than ergodicity. The ergodicity of all powers of $T$ is a sufficient condition $[35]$. 
Proof of Lemma 2.3. The assertion that $\ell(x)$ is invariant for $\operatorname{ergodic}(X, \beta, \mu, T)$ immediately implies $\ell(x)=\int_{X} f d \mu$.

Because $\left|(1 / N) \sum_{n=1}^{N} f\left(T^{a_{n}} x\right)\right| \leq M f(x) \quad(N=1,2, \ldots)$ and $(M f)^{2} \in L^{1}$, the dominated convergence theorem implies that

$$
g(x)=\lim _{N \rightarrow \infty} \frac{1}{N} \sum_{n=1}^{N} f\left(T^{a_{n}} x\right)
$$

exists in $L^{2}$-norm. Lemma 2.1 tells us that $g(x)$ is $T$ invariant if and only if $\left(k_{n}\right)_{n \geq 1}$ is Hartman uniformly distributed.

All we have to do now is to show that the pointwise limit is the same as the norm limit, that is, that $\ell_{T, f}(x)=g(x), \mu$ almost everywhere. We consider a sequence of natural numbers $\left(N_{t}\right)_{t \geq 1}$ such that

$$
\left\|\frac{1}{N_{t}} \sum_{n=1}^{N_{t}} f\left(T^{k_{n}} x\right)-g(x)\right\|_{2} \leq \frac{1}{t} .
$$

Thus

$$
\sum_{t=1}^{\infty} \int_{X}\left|\frac{1}{N_{t}} \sum_{n=1}^{N_{t}} f\left(T^{k_{n}} x\right)-g(x)\right|^{2} d \mu<\infty .
$$

Fatou's lemma tells us that

$$
\int_{X}\left(\sum_{t=1}^{\infty}\left|\frac{1}{N_{t}} \sum_{n=1}^{N_{t}} f\left(T^{k_{n}} x\right)-g(x)\right|^{2}\right) d \mu<\infty,
$$

which implies that

$$
\sum_{t=1}^{\infty}\left|\frac{1}{N_{t}} \sum_{n=1}^{N_{t}} f\left(T^{k_{n}} x\right)-g(x)\right|^{2}<\infty
$$

almost everywhere. This means that

$$
\left|\frac{1}{N_{t}} \sum_{n=1}^{N_{t}} f\left(T^{k_{n}} x\right)-g(x)\right|=o(1),
$$

$\mu$ almost everywhere. As $\left(k_{n}\right)_{n \geq 1}$ is $L^{2}$-good universal we must have $\ell_{T, f}(x)=g(x), \mu$ almost everywhere.

We also use the following lemma from [19], which in the case $k_{n}=n(n=1,2, \ldots)$ is classical and due to Oxtoby [30].

LeMma 2.4. Suppose $\left(k_{n}\right)_{n \geq 1}$ is Hartman uniformly distributed and $L^{\infty}$-good universal. Let $T$ be a continuous map of a compact metrizable space $X$. Also let $\mu$ denote a measure defined on a $\sigma$-algebra $\mathcal{B}$ of subsets of $X$. The following statements are equivalent.

(a) The transformation $(X, \mathcal{B}, \mu, T)$ is uniquely ergodic.

(b) Whenever $f$ is a continuous function on $X$ we have

$$
\lim _{N \rightarrow \infty} \frac{1}{N} \sum_{n=1}^{N} f\left(T^{k_{n}} x\right)=\int_{X} f d \mu
$$

pointwise on $X$, that is, for all $x \in X$. 
Let $\delta_{a}$ denote the Kronecker delta function at $a$. We say that $\left(x_{n}\right)_{n \geq 1} \subseteq X$ for a compact metric space $X$ is asymptotically distributed with respect to a measure $m$ if

$$
\lim _{N \rightarrow \infty} \frac{1}{N} \sum_{n=1}^{N} \delta_{x_{n}}=m
$$

in the weak star limit. For $x \in X$ a sequence of natural numbers $\kappa=\left(k_{n}\right)_{n \geq 1}$ and a measure-preserving transformation $T: X \rightarrow X$ we say that $x$ is $\kappa$-generic with respect to $m$ if $x_{n}=T^{k_{n}} X(n=1,2 \ldots)$ is asymptotically distributed with respect to $m$.

In light of Lemmas 2.3 and 2.4 we see that if $\kappa$ is both Hartman uniformly distributed and $L^{\infty}$ good universal then $\mu$ almost all $x$ are $\kappa$-generic with respect to $\mu$. For the following lemma see [23, pp. 2-7]. For a real number $x$, let $e(x)$ denote $e^{2 \pi i x}$. The next lemma states Weyl's famous criterion on uniform distribution.

LEMMA 2.5. The following are equivalent.

(i) $\left(x_{n}\right)_{n \geq 1}$ is uniformly distributed modulo one.

(ii) We have

$$
\lim _{N \rightarrow \infty} \frac{1}{N} \sum_{n=1}^{N} e\left(h x_{n}\right)=0
$$

for $h \in \mathbb{Z} \backslash\{0\}$.

(iii) For each continuous function $f:[0,1) \rightarrow \mathbb{R}$ we have

$$
\lim _{N \rightarrow \infty} \frac{1}{N} \sum_{n=1}^{N} f\left(x_{n}\right)=\int_{0}^{1} f(x) d x
$$

We have the following lemma of Furstenberg [15].

LEMMA 2.6. Let $T: X \rightarrow X$ be a homeomorphism of a compact metric space that preserves a measure $\mu$ such that $(X, \mathcal{B}, \mu, T)$ is uniquely ergodic. Let $G$ be a compact group with Haar measure $m_{G}$ and let $c: X \rightarrow G$ be a continuous map. Define the skew product map $\mathcal{S}$ on $Y=X \times G$ by $\mathcal{S}(x, g)=(T(x), c(x) g)$. If $\mathcal{S}$ is ergodic with respect to $\mu \times m_{G}$ then it is uniquely ergodic.

We now specialize to the case $G=\mathbb{T}^{l}$. For irrational $\alpha$ and $\mathbf{x}=\left(x_{1}, \ldots, x_{l}\right)$ set $\mathcal{S}(\mathbf{x})=\left(x_{1}+\alpha, x_{2}+x_{1}, \ldots, x_{l}+x_{l-1}\right)$. A standard Fourier series argument shows this map is ergodic. See [38, p.175] for details. Furstenberg's lemma now shows it must be uniquely ergodic. Suppose $\psi_{l}(x):=\psi(x)$ is of degree $l$ and let $\psi_{i-1}(x):=$ $\psi_{i}(x+1)-\psi_{i}(x)(i=2, \ldots, l)$. So $\psi_{1}(x)=\alpha_{l} x+\beta$ for some real number $\beta$. Observe that $\mathcal{S}^{n}\left(\left(\psi_{1}(0), \ldots, \psi_{l}(0)\right)\right)=\left(\psi_{1}(n), \ldots, \psi_{l}(n)\right)(n=1,2, \ldots)$. Using Lemma 2.2 , all points in $\mathbb{T}^{k}$ are $k$-generic if $k$ is good, so, given a continuous function $f: \mathbb{T}^{l} \rightarrow \mathbb{C}$, we have

$$
\lim _{N \rightarrow \infty} \frac{1}{N} \sum_{n=1}^{N} f\left(\psi_{1}\left(k_{n}\right), \ldots, \psi_{l}\left(k_{n}\right)\right)=\int_{\mathbb{T}^{l}} f\left(t_{1}, \ldots, t_{l}\right) d t_{1} \ldots d t_{l} .
$$

Setting $g\left(t_{l}\right)=f\left(t_{1}, \ldots, t_{l}\right)$, we have

$$
\lim _{N \rightarrow \infty} \frac{1}{N} \sum_{n=1}^{N} g\left(\psi_{l}\left(k_{n}\right)\right)=\int_{\mathbb{T}} g\left(t_{l}\right) d t_{l}
$$


for any continuous $g: \mathbb{T} \rightarrow \mathbb{C}$ as required, thus proving Theorem 1.1 by Weyl's criterion on uniform distribution in the special case where $\alpha_{l}$ is irrational.

Now suppose $\alpha_{l}$ is rational. Then $\alpha_{l}=p / q$ where $p, q \in \mathbb{Z}$, the pair $p$ and $q$ are coprime and $q \neq 0$. Also by assumption one of the numbers $\left\{\alpha_{1}, \ldots, \alpha_{l-1}\right\}$ is irrational and we can assume without loss of generality that $l \geq 2$. The latter assumption can be made because the result is immediate for $l=1$ from the the Hartman uniform distribution of the sequence $\left(k_{n}\right)_{n \geq 1}$. To complete the proof of Theorem 1.1 we argue by induction and assume the result is true for all polynomials for $l \leq k$.

Suppose now that $\psi$ has degree $k+1$. Because $\alpha_{l}=p / q$, for each $j=0,1, \ldots, q-1$ the sequence $\left(\left\{\psi\left(k_{n}\right)\right\}\right)_{k_{n} \equiv j \bmod q}$ coincides with $\left(\left\{\phi\left(k_{n}\right)\right\}\right)_{k_{n} \equiv j \bmod q}$ for a polynomial $\phi$ of degree $k$ satisfying the hypothesis of Theorem 1.1. By hypothesis we know $\left(\left\{\phi\left(k_{n}\right)\right\}\right)_{n \geq 1}$ is uniformly distributed modulo one. We wish to deduce that $\left(\left\{\phi\left(k_{n}\right)\right\}\right)_{k_{n} \equiv j \bmod q}$ is uniformly distributed modulo one. Unlike in the case when $k_{n}=n(n=1,2, \ldots)$, where this is immediate from the uniform distribution of $(\{\phi(n)\})_{n \geq 1}$, for general $\left(k_{n}\right)_{n \geq 1}$ a little more care is needed. Let $k(N)$ denote the number of elements of $\left(k_{n}\right)_{n \geq 1}$ in the interval $[1, N]$. By Weyl's criterion, which is Lemma 2.5(ii), we have to show for each $h \in \mathbb{Z}$ that

$$
\lim _{N \rightarrow \infty} \frac{1}{k(N)} \sum_{1 \leq k_{n} \leq N ; k_{n} \equiv j \bmod q} e\left(h \phi\left(k_{n}\right)\right)=0 .
$$

Let $G_{q}$ denote the group of residues modulo $q$ and let $\hat{G}_{q}$ its dual group. Using the orthogonality property on this group, we have

$$
I(n, j, q):=\frac{1}{q} \sum_{\chi \in \hat{G}_{q}} \chi\left(k_{n}\right) \bar{\chi}(j)= \begin{cases}1 & \text { if } k_{n} \equiv j \bmod q, \\ 0 & \text { otherwise. }\end{cases}
$$

Note that the function $I(n, j, q)$ is the indicator function of the residue class $j$ modulo $q$. This means that

$$
\begin{aligned}
& \lim _{N \rightarrow \infty} \frac{1}{k(N)} \sum_{1 \leq k_{n} \leq N ; k_{n} \equiv j \bmod q} e\left(h \phi\left(k_{n}\right)\right) \\
& \quad=\lim _{N \rightarrow \infty} \frac{1}{k(N)} \sum_{1 \leq k_{n} \leq N} e\left(h \phi\left(k_{n}\right)\right)\left(\frac{1}{q} \sum_{\chi \in \hat{G}_{q}} \chi\left(k_{n}\right) \bar{\chi}(j)\right) .
\end{aligned}
$$

Rearranging the double sum, we have that

$$
\begin{aligned}
\frac{1}{k(N)} & \sum_{1 \leq k_{n} \leq N} e\left(h \phi\left(k_{n}\right)\right)\left(\frac{1}{q} \sum_{\chi \in \hat{G}_{q}} \chi\left(k_{n}\right) \bar{\chi}(j)\right) \\
= & \frac{\bar{\chi}(j)}{q} \sum_{\chi \in \hat{G}_{q}}\left(\frac{1}{k(N)} \sum_{1 \leq k_{n} \leq N} e\left(h \phi\left(k_{n}\right)\right) \chi\left(k_{n}\right)\right) .
\end{aligned}
$$

Notice that the group $\hat{G}_{q}$ coincides with the group of roots of unity on the unit circle in the complex plane. This means that $\chi\left(k_{n}\right)=e^{2 \pi i k_{n}(s / t)}$ for some $s, t \in \mathbb{Z}$ with $t$ dividing $q$. Moreover, $s$ and $t$ are coprime. This means that 


$$
\begin{aligned}
\frac{\bar{\chi}(j)}{q} & \sum_{\chi \in \hat{G}_{q}}\left(\frac{1}{k(N)} \sum_{1 \leq k_{n} \leq N} e\left(h \phi\left(k_{n}\right)\right) \chi\left(k_{n}\right)\right) \\
= & \frac{\bar{\chi}(j)}{q} \sum_{\chi \in \hat{G}_{q}}\left(\frac{1}{k(N)} \sum_{1 \leq k_{n} \leq N} e\left(h \phi^{\prime}\left(k_{n}\right)\right)\right),
\end{aligned}
$$

where the polynomial $\phi^{\prime}$ coincides with the polynomial $\phi$ except that the coefficient $\alpha_{1}$ is replaced by the coefficient $\alpha_{1}-s / t h$. Note that by the inductive hypothesis we know that $\left(\phi^{\prime}\left(k_{n}\right)\right)_{n=1}^{\infty}$ is uniformly distributed modulo one. Thus, using Weyl's criterion, $\left(\left\{\phi\left(k_{n}\right)\right\}\right)_{k_{n}} \equiv j \bmod q$ is uniformly distributed modulo one, implying $\left(\left\{\psi\left(k_{n}\right)\right\}\right)_{k_{n} \equiv j \bmod q}$ is uniformly distributed modulo one, which finally implies $\left(\left\{\psi\left(k_{n}\right)\right\}\right)_{n=1}^{\infty}$ is uniformly distributed modulo one as required, so Theorem 1.1 is proved.

It is natural to ask if the assumption of $\left(k_{n}\right)_{n \geq 1}$ being good can be replaced by the strictly weaker assumption that $\left(k_{n}\right)_{n \geq 1}$ is Hartman uniformly distributed on $\mathbb{Z}$. The usual differencing argument used to prove Weyl's theorem does not seem to be sufficient.

\section{Proof of Corollary 1.2}

For coprime integers $p, q$ with $q$ non-zero let

$$
G(p, q, \phi)=\lim _{N \rightarrow \infty} \frac{1}{N} \sum_{j=1}^{N} e\left(\frac{p}{q} \phi\left(k_{j}\right)\right),
$$

which must exist as $\left(k_{j}\right)_{j \geq 1}$ is uniformly distributed on $\mathbb{Z}$. Using Theorem 1 .1, we have

$$
\lim _{N \rightarrow \infty} \frac{1}{N} \sum_{n=0}^{N-1} e\left(\phi\left(k_{n}\right) \theta\right)= \begin{cases}0 & \text { if } \theta \notin \mathbb{Q}, \\ G(p, q, \phi) & \text { if } \theta=\frac{p}{q} .\end{cases}
$$

Let $U f(x)=f(T x)$. This is a unitary operator on $L^{2}$ as $T$ is measure-preserving. Also let $U^{-1}$ denote the $L^{2}$ adjoint of $U$. Recall that we say that a sequence $\left(c_{n}\right)_{n \in \mathbb{Z}}$ is positive definite if, given a bi-sequence of complex numbers $\left(z_{n}\right)_{n \in \mathbb{Z}}$, only finitely many of whose terms are non-zero, we have $\sum_{n, m \in \mathbb{Z}} c_{n-m} z_{n} \overline{z_{m}} \geq 0$. Here $\bar{z}$ is the conjugate of the complex number $z$. Let $\langle f, g\rangle=\int_{X} f \bar{g} d \mu$ (i.e. the standard inner product on $L^{2}$ ). Notice that $\left(\left\langle U^{n} f, f\right\rangle\right)_{n \in \mathbb{Z}}$ is positive definite. Recall that the Bochner-Herglotz theorem [21] says that there is a measure $\omega_{f}$ on $\mathbb{T}$ such that

$$
\left\langle U^{n} f, f\right\rangle=\int_{\mathbb{T}} z^{n} d \omega_{f}(z) \quad(n \in \mathbb{Z}) .
$$

From this we see that

$$
\begin{aligned}
& \left\|\frac{1}{N} \sum_{n=0}^{N-1} f\left(T^{\phi\left(k_{n}\right)} x\right)-\frac{1}{M} \sum_{n=0}^{M-1} f\left(T^{\phi\left(k_{n}\right)} x\right)\right\|_{2}^{2} \\
& \quad=\int_{\mathbb{T}}\left|\frac{1}{N} \sum_{n=0}^{N-1} e\left(\phi\left(k_{n}\right) \theta\right)-\frac{1}{M} \sum_{n=0}^{M-1} e\left(\phi\left(k_{n}\right) \theta\right)\right|^{2} d \omega_{f}(\theta) .
\end{aligned}
$$

Using Theorem 1.1 and Weyl's criterion, this can be as small as you like by choosing $M$ and $N$ large enough. This means that $\left((1 / N) \sum_{n=0}^{N-1} f\left(T^{\phi\left(k_{n}\right)} x\right)\right)_{N \geq 1}$ is a Cauchy sequence in $L^{2}$, proving Corollary 1.2. 


\section{Proof of Corollaries 1.3 and 1.4}

Now for each natural number $N$, the complex function $(1 / N) \sum_{n=1}^{N} z^{n}$ is equal to 1 for all $N$ if $z=1$, and it tends to 0 for all other $z$ such that $z$ is of absolute value 1 as $N$ tends to infinity. This means that if

$$
A_{N} f(x)=\frac{1}{N} \sum_{n=1}^{N} f\left(T^{n} x\right) \quad(N=1,2, \ldots),
$$

then $\left\langle A_{N} f, f\right\rangle=\int_{\mathbb{T}}\left((1 / N) \sum_{n=1}^{N} e(n \theta)\right) d \omega_{f}(\theta)$ tends to $\omega_{f}(\{0\})$ as $N$ tends to infinity. By the mean ergodic theorem, however, for $f$ in $L^{2}$, if $P_{T} f$ is the projection of $f$ onto the $T$ invariant subspace of $L^{2}$ then $A_{N} f$ tends to $P_{T} f$ in $L^{2}$-norm as $N$ tends to infinity. This means that $\left\langle P_{T} f, f\right\rangle=w_{f}(\{0\})$ and so, by Cauchy's inequality,

$$
\omega_{f}(\{0\})=\left\langle P_{T} f, f\right\rangle=\left\langle P_{T} f, P_{T} f\right\rangle \geq\left|\int P_{T} f d \mu\right|^{2}=\left|\int_{X} f d \mu\right|^{2} .
$$

Let $\phi$ denote a non-constant polynomial mapping the natural numbers to themselves such that $\phi(0)=0$. Let $L_{s, n}=\left\{\phi\left(s^{*} k\right): k \in \kappa \cap[1, n](n=1,2 \ldots)\right.$ where $s^{*}$ is the least common multiple of the first $s$ integers $\}$, let $F_{k}=\{a / q: 1 \leq a<q \leq k ;(a, q)=1\}$, let $F_{k}^{c}$ denote its complement in the rational numbers and let $\omega_{f}=\omega_{i}+\omega_{r}$ where $\omega_{r}$ are the atoms of $\omega_{f}$ on the rationals. Then for arbitrary $k_{0}$ we have

$$
\begin{aligned}
\frac{1}{\left|L_{k_{0}, n}\right|} \sum_{v \in L_{k_{0}, n}}\left\langle U^{v} f, f\right\rangle= & \omega_{f}(\{0\})+\sum_{\theta \in F_{k_{0}}} \omega_{r}(\{\theta\}) \\
& +\sum_{\theta \in F_{k_{0}}^{c}} \omega_{r}(\{\theta\})\left(\frac{1}{\left|L_{k_{0}, n}\right|} \sum_{v \in L_{k_{0}, n}} e(v \theta)\right) \\
& +\int_{\mathbb{T}}\left(\frac{1}{\left|L_{k_{0}, n}\right|} \sum_{v \in L_{k_{0}, n}} e(v \alpha)\right) d \omega_{i}(\alpha) .
\end{aligned}
$$

The second term on the right-hand side of this identity is non-negative, the third becomes less than $\epsilon$ for large enough $k_{0}$, and the final term tends to zero as $n$ tends to infinity by Theorem 1.1 and Weyl's criterion, so, choosing $f=\chi_{A}$ with $\mu(A)>0$, we have

$$
\limsup _{n \rightarrow \infty} \frac{1}{\left|L_{k_{0}, n}\right|} \sum_{v \in L_{k_{0}, n}} \mu\left(A \cap T^{-v} A\right) \geq \mu^{2}(A)-\epsilon,
$$

as required.

\section{Proof of Corollary 1.5}

We begin with some technical results we will need later. The first is abstracted from special cases dealt with in [2].

LEMMA 5.1. Given $\epsilon>0$, if $X=\left\{x_{1}, \ldots, x_{s}\right\}$ is any set of finitely many points contained in $\mathbb{T}$ such that for every natural number $n$ indexing the sequence $\left(a_{n}\right)_{n \geq 1}$ the dilation $a_{n} X$ is not $\epsilon$ dense, then there is an absolute constant $C>0$ such that if

$$
M=\left[\left(\frac{1}{\epsilon}\right) \log ^{2}\left(\frac{1}{\epsilon}\right)\right]
$$


then we have

$$
s^{2} \leq\left(\frac{C}{\epsilon}\right) \sum_{m=1}^{M} \sum_{j=1}^{s} \sum_{l=1}^{s} \frac{1}{N} \sum_{n=1}^{N} e_{m}\left(a_{n}\left(x_{j}-x_{l}\right)\right),
$$

where $e_{m}(t)=e^{2 \pi i m t}$.

We next need the following estimate for rational exponential sums which is due to Hua [18].

LEMMA 5.2. Let $\theta$ denote a polynomial of degree $L$ mapping the natural numbers to themselves. Then if $\epsilon_{1}>0$, there is a constant $C>0$ such that

$$
\left|\frac{1}{b} \sum_{a=1}^{b} e_{1}\left(\frac{\theta(a)}{b}\right)\right| \leq \frac{C}{b^{1 / L-\epsilon_{1}}} .
$$

The following is also taken from [2].

LEMMA 5.3. Let $\left\{x_{1}, \ldots, x_{s}\right\}$ be an arbitrary set of $s$ distinct points in the unit interval $[0,1)$. Denote by $h_{m}$ the number of pairs $(i, j)$ with $1 \leq i<j \leq s$, such that $m\left(x_{i}-x_{j}\right)$ is an integer. Suppose $\beta>0$. Then if $s$ is sufficiently large for any $m \geq 1$, the partial sum

$$
H_{m}=\sum_{l=1}^{m} h_{l}
$$

satisfies the inequality

$$
H_{m} \leq(s m)^{\beta+1} .
$$

The trivial bound here is $H_{m} \leq s m^{2}$.

Following Lemma 5.1, given $\epsilon>0$ and a finite set $X=\left\{x_{1}, \ldots, x_{s}\right\}$ contained in $\mathbb{T}$, if the dilation $\phi\left(k_{n}\right) X$ is not $\epsilon$ dense, setting

$$
M=\left[\left(\frac{1}{\epsilon}\right) \log ^{2}\left(\frac{1}{\epsilon}\right)\right]
$$

we have

$$
s^{2} \leq\left(\frac{C}{\epsilon}\right) \sum_{m=1}^{M} \sum_{j=1}^{s} \sum_{l=1}^{s} \frac{1}{N} \sum_{n=1}^{N} e_{m}\left(\phi\left(k_{n}\right)\left(x_{j}-x_{l}\right)\right) .
$$

As a consequence of Theorem 1.1 and Weyl's criterion, as $N$ tends to infinity, when for a particular $j$ and $l$ the difference $x_{j}-x_{k}$ is irrational the average furthest to the right in (5.1) tends to zero. This means that in estimating the right-hand side of (5.1) we need only consider the contribution of the terms in the double sum in $j$ and $l$ for which the corresponding $x_{j}-x_{l}$ is rational.

Now for rational $a / b$, in reduced form we may clearly write

$$
m \phi(n) \frac{a}{b}=\frac{\left(a_{l}^{\prime} n^{l}+\cdots+a_{0}^{\prime}\right)}{b^{\prime}}=\frac{\theta(n)}{b^{\prime}}
$$

where the highest common factor of the integers $a_{l}^{\prime}, \ldots, a_{0}^{\prime}$, and $b^{\prime}$ is $1, b^{\prime}$ denotes $b /(m, b)$, and $(m, b)$ denotes the highest common factor of $m$ and $b$. Because $\left(k_{n}\right)_{n=1}^{\infty}$ 
is uniformly distributed among the residue classes modulo $b^{\prime}$, and further because of the fact that the value of $m \phi\left(k_{n}\right)(a / b)$ is $\theta(c) / b^{\prime}$ when $k_{n} \equiv c\left(\bmod b^{\prime}\right)$, if $x_{j}-x_{k}=a / b$, we have

$$
\lim _{N \rightarrow \infty} \frac{1}{N} \sum_{n=1}^{N} e_{m}\left(\phi\left(k_{n}\right)\left(x_{j}-x_{l}\right)\right)=\frac{1}{b^{\prime}} \sum_{a=1}^{b^{\prime}} e_{1}\left(\frac{\theta(a)}{b^{\prime}}\right) .
$$

From Lemma 5.2 we have that

$$
\left|\frac{1}{b^{\prime}} \sum_{a=1}^{b^{\prime}} e_{1}\left(\frac{\theta(a)}{b^{\prime}}\right)\right|<\frac{C}{\left(b^{\prime}\right)^{1 / l-\epsilon_{1}}} \leq C\left(\frac{(m, b)}{b}\right)^{1 / l-\epsilon_{1}} .
$$

Also because there are at most $M / r$ multiples of $r$ that divide $b$ less than $M$ we know that

$$
\sum_{m=1}^{M}(m, b)^{1 / l-\epsilon_{1}} \leq \sum_{\substack{r \mid b \\ r \leq M}}\left(\frac{M}{r}\right) r^{1 / l-\epsilon_{1}}
$$

We also note that

$$
\sum_{\substack{r \mid b \\ r \leq M}}\left(\frac{M}{r}\right) r^{1 / l-\epsilon} \leq M d(b),
$$

where $d(n)$ denotes the number of integers between 1 and $n$ inclusive that there are that divide $n$. Note that, given $\epsilon_{2}>0$, we have $d(n)=o\left(n^{\epsilon_{2}}\right)$. From this (5.2) tells us that

$$
\sum_{m=1}^{M}\left|\lim _{N \rightarrow \infty} \frac{1}{N} \sum_{n=1}^{N} e_{m}\left(\phi\left(k_{n}\right)\left(x_{j}-x_{l}\right)\right)\right| \leq C M b^{-1 / l+\epsilon_{1}+\epsilon_{2}} .
$$

Let

$$
g_{b}=\#\left\{(j, k): 1 \leq j<l \leq s: x_{j}-x_{l}=\frac{a}{b} \text { for some } a ;(a, b)=1\right\}
$$

and let

$$
G_{b}=\sum_{i=1}^{b} g_{i}
$$

Then, setting $\epsilon=\epsilon_{1}+\epsilon_{2}$ and using partial summation, we have

$$
\begin{aligned}
s^{2} & \leq C\left(\frac{M}{\epsilon}\right)\left(s+\sum_{b \geq 2} g_{b} b^{-1 / l+\epsilon}\right) \\
& =C\left(\frac{M}{\epsilon}\right)\left(s+\sum_{b \geq 2} G_{b}\left(b^{-1 / l+\epsilon}-(b+1)^{-1 / l+\epsilon}\right)\right) .
\end{aligned}
$$

From the trivial estimate in Lemma 5.3 we see that we have $G_{b} \leq s^{2}$ for $b \geq s$. If $b<s$ we know $G_{b} \leq H_{b} \leq s b^{1+\beta}$. Therefore the above expression is majorized by

$$
\begin{gathered}
C\left(\frac{M}{\epsilon}\right)\left(s+\sum_{b \geq 2}(s b)^{1+\beta} b^{-1 / l+\epsilon}+s^{2} s^{-1 / l+\epsilon}\right) \\
\leq C\left(\frac{M}{\epsilon}\right) s^{1+\beta}\left(\sum_{b=1}^{s} b^{2 \beta-1 / l}+s^{1-1 / l}\right)
\end{gathered}
$$




$$
\leq C\left(\frac{M}{\epsilon}\right) s^{2+3 \beta-1 / l}\left(1+s^{-2 \beta}\right) .
$$

Given our value for $M$, this tells us that

$$
s \leq\left(\frac{1}{\epsilon}\right)^{2+\delta},
$$

as required.

\section{List of known good universal sequences}

In this section we give some examples of $L^{p}$-good universal sequences for some $p \geq 1$. Examples 1 and 3-6 are Hartman uniformly distributed. Example 2 is not Hartman uniformly distributed in general.

(1) The natural numbers. The sequence $(n)_{n=1}^{\infty}$ is $L^{1}$-good universal. This is Birkhoff's pointwise ergodic theorem.

(2) Polynomial-like sequences. If $\phi(x)$ is a polynomial such that $\phi(\mathbb{N}) \subseteq \mathbb{N}$ and $p>1$, then $(\phi(n))_{n=1}^{\infty}$ and $\left(\phi\left(p_{n}\right)\right)_{n=1}^{\infty}$, where $p_{n}$ is the $n$th prime, are $L^{p}$-good universal sequences. See [13] and [25], respectively.

(3) Condition H. Sequences $\left(k_{n}\right)_{n=1}^{\infty}$ that are both $L^{p}$-good universal and Hartman uniformly distributed can be constructed as follows. Set $k_{n}=[\tau(n)](n=1,2, \ldots)$, where $\tau:[1, \infty) \rightarrow[1, \infty)$ is a differentiable function whose derivative increases with its argument. Let $\Omega_{m}$ denote the cardinality of the set $\left\{n: a_{n} \leq m\right\}$, and suppose, for some function $\varphi:[1, \infty) \rightarrow[1, \infty)$ increasing to infinity as its argument does, that we set

$$
\varrho(m)=\sup _{\{z\} \in[1 / \varphi(m), 1 / 2)}\left|\sum_{n: k_{n} \leq m} e\left(z k_{n}\right)\right| .
$$

Suppose also, for some decreasing function $\rho:[1, \infty) \rightarrow[1, \infty)$ and some positive constant $\omega>0$, that

$$
\frac{\varrho(m)+\Omega_{[\varphi(m)]}+m / \varphi(m)}{\Omega_{m}} \leq \omega \rho(m) .
$$

Then if we have

$$
\sum_{n=1}^{\infty} \rho\left(\theta^{n}\right)<\infty
$$

for all $\theta>0$, we say that $\left(a_{n}\right)_{n=1}^{\infty}$ satisfies condition $\mathrm{H}$, see $[\mathbf{2 7}, \mathbf{3 6}]$.

Sequences satisfying condition $\mathrm{H}$ are known to be both Hartman uniformly distributed and $L^{p}$-good universal. Specific sequences of integers that satisfy condition $\mathrm{H}$ include $a_{n}=[\tau(n)](n=1,2, \ldots)$ where:

(I) $\quad \tau(n)=n^{\gamma}$ if $\gamma>1$ and $\gamma \notin \mathbb{N}$.

(II) $\tau(n)=e^{\log ^{\gamma} n}$ for $\gamma \in\left(1, \frac{3}{2}\right)$.

(III) $\tau(n)=b_{k} n^{k}+\cdots+b_{1} n+b_{0}$ for $b_{k}, \ldots, b_{1}$ not all rational multiplies of the same real number.

(IV) Hardy fields. By a Hardy field, we mean a closed subfield (under differentiation) of the ring of germs at $+\infty$ of continuous real-valued functions with addition and multiplication taken to be pointwise. Let $\mathcal{H}$ denote the union of all Hardy fields. 
Conditions for $\left(a_{n}\right)_{n=1}^{\infty}=([\psi(n)])_{n=1}^{\infty}$, where $\psi \in \mathcal{H}$, to satisfy condition $\mathrm{H}$ are given by the hypotheses of [12, Theorems 3.4, 3.5 and 3.8]. Note that the term ergodic is used in this paper in place of the older term Hartman uniformly distributed.

(4) A random example. Suppose that $S=\left(k_{n}\right)_{n=1}^{\infty}$ is a strictly increasing sequence of natural numbers. By identifying $S$ with its characteristic function $\chi_{S}$, we may view it as a point in $\Lambda=\{0,1\}^{\mathbb{N}}$, the set of maps from $\mathbb{N}$ to $\{0,1\}$. We may endow $\Lambda$ with a probability measure by viewing it as a Cartesian product $\Lambda=\prod_{n=1}^{\infty} X_{n}$, where, for each natural number $n$, we have $X_{n}=\{0,1\}$ and specify the probability $v_{n}$ on $X_{n}$ by $v_{n}(\{1\})=\omega_{n}$ with $0 \leq \omega_{n} \leq 1$ and $v_{n}(\{0\})=1-\omega_{n}$ such that $\lim _{n \rightarrow \infty} \omega_{n} n=\infty$. The desired probability measure on $\Lambda$ is the corresponding product measure $v=$ $\prod_{n=1}^{\infty} v_{n}$. The underlying $\sigma$-algebra $\mathcal{A}$ is that generated by the cylinders

$$
\left\{\left(\Delta_{n}\right)_{n=1}^{\infty} \in \Lambda: \Delta_{n_{1}}=\alpha_{n_{1}}, \ldots, \Delta_{n_{k}}=\alpha_{n_{k}}\right\}
$$

for all possible choices of $n_{1}, \ldots, n_{k}$ and $\alpha_{n_{1}}, \ldots, \alpha_{n_{k}}$. Then almost every point $\left(a_{n}\right)_{n=1}^{\infty}$ in $\Lambda$, with respect to the measure $v$, is Hartman uniformly distributed. See [13, Proposition 8.2 (i)] for the details of this. Again Hartman uniformly distributed sequences are called ergodic sequences in this paper.

(5) Block sequences. Suppose that $\left(a_{n}\right)_{n=1}^{\infty}=\bigcup_{n=1}^{\infty}\left[d_{n}, e_{n}\right]$ is ordered by absolute value for disjoint $\left(\left[d_{n}, e_{n}\right]\right)_{n=1}^{\infty}$ with $d_{n-1}=O\left(e_{n}\right)$ as $n$ tends to infinity. Note that this allows the possibility that $\left(a_{n}\right)_{n=1}^{\infty}$ is zero density. This example is an immediate consequence of Tempelman's semigroup ergodic theorem. See [4, p. 218]. Being a group average ergodic theorem, this pointwise limit must be invariant, which ensures that the block sequence must be Hartman uniformly distributed. The proof of this, which we do not need in this paper and is hence omitted, is a simple exercise in spectral theory.

(6) Random perturbation of good sequences. Suppose that $\left(a_{n}\right)_{n=1}^{\infty}$ is an $L^{p}$-good universal sequence which is also Hartman uniformly distributed. Let $\theta=\left(\theta_{n}\right)_{n=1}^{\infty}$ be a sequence of $\mathbb{N}$-valued independent, identically distributed random variables with basic probability space $(Y, \mathcal{A}, \mathcal{P})$, and a $\mathcal{P}$-complete $\sigma$-field $\mathcal{A}$. Let $\mathbb{E}$ denote expectation with respect to the basic probability space $(Y, \mathcal{A}, \mathcal{P})$. Assume that there exist $0<\alpha<1$ and $\beta>1 / \alpha$ such that

$$
a_{n}=O\left(e^{n^{\alpha}}\right) \text { and } \mathbb{E} \log _{+}^{\beta}\left|\theta_{1}\right|<\infty .
$$

Then $\left(k_{n}+\theta_{n}(\omega)\right)_{n=1}^{\infty}$ is both $L^{p}$-good universal and Hartman uniformly distributed [29].

Acknowledgements. We thank the referee for very detailed comments which materially improved the presentation of this paper.

\section{REFERENCES}

[1] M. Ajtai, I. Havas and J. Komlós. Every group admits a bad topology. Studies in Pure Mathematics. Eds. P. Erdös, L. Alpár, G. Halász and A. Sárközy. Birkhäuser, Basel, 1983, pp. 21-34. 
[2] N. Alon and Y. Peres. Uniform dilations. Geom. Funct. Anal. 2(1) (1992), 1-28.

[3] N. H. Asmar and R. Nair. Certain averages on the a-adic numbers. Proc. Amer. Math. Soc. 114(1) (1992), 21-28.

[4] A. Bellow and V. Losert. On sequences of zero density in ergodic theory. Conference in Modern Analysis and Probability (New Haven, CT 1982) (Contemporary Mathematics, 26). American Mathematical Society, Providence, RI, 1984, pp. 49-60.

[5] D. Berend and Y. Peres. Asymptotically dense dilations of sets on the circle. J. Lond. Math. Soc. (2) 47(1) (1993), 1-17.

[6] V. Bergelson, G. Kolesnik, M. Madritsch, Y. Son and R. Tichy. Uniform distribution of prime powers and sets of recurrence and van der Corput sets in $\mathbb{Z}^{k}$. Israel J. Math. 201(2) (2014), 729-760.

[7] V. Bergelson and A. Leibman. A Weyl-type equidistribution theorem in finite characteristic. Adv. Math. 289 (2016), 928-950.

[8] V. Bergelson and E. Lesigne. Van der Corput sets in $\mathbb{Z}^{d}$. Colloq. Math. 110(1) (2008), 1-49.

[9] A. Bertrand-Mathis. Ensembles intersectifs et récurrence de Poincaré. Israel J. Math. 55(2) (1986), 184-198.

[10] J. Blum and B. Eisenberg. Generalized summing sequences and the mean ergodic theorem. Proc. Amer. Math. Soc. 42 (1974), 423-429.

[11] M. Boshernitzan. Homogeneously distributed sequences and Poincaré sequences of integers of sublacunary growth. Monatsh. Math. 96(3) (1983), 173-181.

[12] M. Boshernitzan, G. Kolesnik, A. Quas and M. Wierdl. Ergodic averaging sequences. J. Anal. Math. 95 (2005), 63-103.

[13] J. Bourgain. On the maximal ergodic theorem for certain subsets of the integers. Israel J. Math. 61(1) (1988), 39-72.

[14] M. Drmota and R. F. Tichy. Sequences, Discrepancies and Applications (Lecture Notes in Mathematics, 1651). Springer, Berlin, 1997.

[15] H. Furstenberg. Strict ergodicity and transformation of the torus. Amer. J. Math. 83 (1961), 573-601.

[16] H. Furstenberg. Poincaré recurrence and number theory. Bull. Amer. Math. Soc. (N.S.) 5(3) (1981), 211-234.

[17] S. Glasner. Almost periodic sets and measures on the Torus. Israel J. Math. 32(2-3) (1979), 161-172.

[18] L. K. Hua. Sur une somme exponentielle. C. R. Acad. Sci. 210 (1940), 520-523.

[19] A. Jaššová, P. Lertchoosakul and R. Nair. On variants of the Halton sequence. Monatsh. Math. 180(4) (2016), 743-764.

[20] H. Kamarul Haili and R. Nair. On certain Glasner sets. Proc. Roy. Soc. Edinburgh Sect. A 133(4) (2003), 849-853.

[21] Y. Katznelson. An Introduction to Harmonic Analysis. Dover, New York, 1976.

[22] S. Kristensen, A. Jaššová, P. Lertchoosakul and R. Nair. On recurrence in positive characteristic. Indag. Math. (N.S.) 26(2) (2015), 346-354.

[23] L. Kuipers and H. Niederreiter. Uniform Distribution of Sequences. Wiley, New York, 1974.

[24] R. Nair. On certain solutions of the Diophantine equation $x-y=p(z)$. Acta Arith. 62(1) (1992), 61-71.

[25] R. Nair. On polynomials in primes and J. Bourgain's circle method approach to ergodic theorems II. Studia Math. 105(3) (1993), 207-233.

[26] R. Nair. On uniformly distributed sequences of integers and Poincaré recurrence. Indag. Math. (N.S.) 9(1) (1998), 55-63.

[27] R. Nair. On uniformly distributed sequences of integers and Poincaré recurrence II. Indag. Math. (N.S.) 9(3) (1998), 405-415.

[28] R. Nair and S. L. Velani. Glasner sets and polynomials in primes. Proc. Amer. Math. Soc. 126(10) (1998), 2835-2840.

[29] R. Nair and M. Weber. On random perturbation of some intersective set. Indag. Math. (N.S) 15(3) (2004), 373-381.

[30] J. C. Oxtoby. Ergodic sets. Bull. Amer. Math. Soc. 58 (1952), 116-136.

[31] A. Sárközy. On difference sets of sequences of integers. I. Acta Math. Acad. Sci. Hungar. 31(1-2) (1978), 125-149.

[32] A. Sárközy. On difference sets of sequences of integers. III. Acta Math. Acad. Sci. Hungar. 31(3-4) (1978), 355-386.

[33] A. Sárközy. On difference sets of sequences of integers. II. Ann. Univ. Sci. Budapest. Eötvös Sect. Math. 21 (1978), 45-53 (1979).

[34] S. Sawyer. Maximal inequalities of weak type. Ann. of Math. (2) 84 (1966), 157-174.

[35] J. P. Thouvenot. La convergence presque sûre des moyennes ergodiques suivant certaines sous-suites d'entiers (d'après Jean Bourgain), Séminaire Bourbaki, Vol. 1989/90. Astérisque 189-190 (1990), exp. no. $719,133-153$. 
[36] R. Urban and J. Zienkiewicz. Weak type $(1,1)$ estimates for a class of discrete rough maximal functions. Math. Res. Lett. 14(2) (2007), 227-237.

[37] R. C. Vaughan. The Hardy-Littlewood Method (Cambridge Tracts in Mathematics, 125), 2nd edn. Cambridge University Press, Cambridge, 1997.

[38] M. Viana and K. Oliveira. Foundations of Ergodic Theory (Cambridge Studies in Advanced Mathematics, 151). Cambridge University Press, Cambridge, 2016.

[39] H. Weyl. Über die Gleichverteilung von Zahlen mod. Eins. Math. Ann. 77(1) (1916), 313-352, Issue 3. 